\title{
Direct hadron production in hadronic collisions
}

\author{
François Arleo ${ }^{\mathrm{a}, *}$, Stanley J. Brodsky ${ }^{\mathrm{b}, \mathrm{c}}$, Dae Sung Hwang ${ }^{\mathrm{d}}$, Anne M. Sickles ${ }^{\mathrm{e}}$ \\ ${ }^{a}$ Laboratoire d'Annecy-le-Vieux de Physique Théorique (LAPTH), UMR5108, Université de Savoie, \\ CNRS, BP 110, 74941 Annecy-le-Vieux cedex, France \\ ${ }^{b}$ SLAC National Accelerator Laboratory, Stanford University, Stanford, California 94309, USA \\ ${ }^{c}$ CP3-Origins, University of Southern Denmark, Odense 5230 M, Denmark \\ ${ }^{d}$ Department of Physics, Sejong University, Seoul 143-747, Korea \\ ${ }^{e}$ Brookhaven National Laboratory, Upton, New York 11973, USA
}

\begin{abstract}
The scaling behavior of the invariant production cross section is a powerful tool in order to probe the dynamics of particle production. Here, we investigate the scaling properties of large- $p_{\perp}$ hadron, jet and prompt photon production in hadronic collisions by comparing systematically world data to NLO QCD predictions. In the hadron sector a significant discrepancy is reported, while prompt photon and jet production data prove in agreement with leadingtwist expectations. We interpret these results as coming from a non-negligible contribution of higher-twist processes, in which the hadron is produced directly in the hard subprocess. Predictions at RHIC are successfully compared to PHENIX preliminary measurements and LHC predictions are given.
\end{abstract}

Keywords: hadron production, higher twist, hadronic colliders

\section{Introduction}

The most important discriminant of the twist of a perturbative QCD subprocess in a hard hadronic collision is the scaling of the inclusive invariant cross section [1],

$$
\sigma^{\mathrm{inv}} \equiv E \frac{\mathrm{d} \sigma}{\mathrm{d}^{3} p}(A B \rightarrow C \mathrm{X})=\frac{F\left(x_{\perp}, \vartheta\right)}{p_{\perp}^{n}},
$$

at fixed $x_{\perp}=2 p_{\perp} / \sqrt{s}$ and center-of-mass (CM) angle $\vartheta$. In the original parton model the power fall-off is simply $n=4$ since the underlying $2 \rightarrow 2$ subprocess amplitude for point-like partons is scale invariant, and there is no dimensionful parameter as in a conformal theory. However, in general additional higher-twist (HT) contributions involving a larger number of elementary fields contributing to the hard subprocess, $n_{\text {active }}>4$, are also expected. For example, the detected hadron $C$ can be produced directly in the hard subprocess, as in an exclusive reaction, and not by the collinear fragmentation of quarks and gluons in the leading twist (LT) approximation; see the illustration in Fig. 1. Unlike quark or gluon fragmentation, the direct processes do not waste sameside energy, thus involving minimal values of the momentum fractions $x_{1}$ and $x_{2}$ where parton distributions

\footnotetext{
*Speaker

Email address: arleo@lapp.in2p3.fr (François Arleo)
}

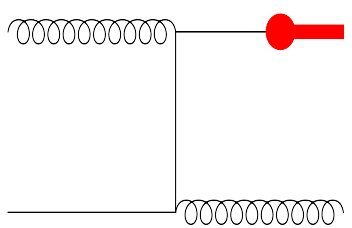

(a)

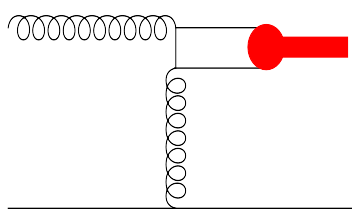

(b)
Figure 1: An example of (a) LT $\left(n_{\text {active }}=4\right)$ and (b) HT $\left(n_{\text {active }}=5\right)$ contributions to large- $p_{\perp}$ meson production

are maximal. Neglecting scaling violations in QCD, the scaling exponent $n$ is given by $n=2 n_{\text {active }}-4$ [1]

The idea of direct hadron production was first considered in the 1970's to explain the large fixed- $x_{\perp}$ scaling exponents reported at ISR and fixed target FNAL energies [1]. However, there has been no comprehensive and quantitative analysis of the data up to now which could bring compelling evidence for such higher-twist effects. In this talk we summarize the novel aspects discussed in our recent analysis [2], namely:

(i) a dedicated analysis of the most recent FNAL, RHIC and Tevatron data on large- $p_{\perp}$ hadrons, prompt photons and jets;

(ii) the systematic comparison of the experimental scaling exponents with NLO QCD expectations;

Preprint submitted to Nuc. Phys. (Proc. Suppl.) 
(iii) predictions at the top RHIC energy and at the LHC.

\section{Analysis}

The exponent $n^{\mathrm{NLO}}$ of mid-rapidity particle production has been computed in QCD at next-to-leading order (NLO) accuracy from Ref. [3]. In Fig. 2, the $x_{\perp}-$ dependence of $n^{\mathrm{NLO}}$ at fixed $p_{\perp}$ has been determined for various hadron species $(\pi, K, p / \bar{p})$. At $p_{\perp}=10 \mathrm{GeV}$ the exponents increase slowly from $n^{\mathrm{NLO}} \simeq 5$ at small values of $x_{\perp}\left(x_{\perp}=10^{-2}\right)$ up to $n^{\mathrm{NLO}} \simeq 6$ at $x_{\perp}=0.5$ with almost no dependence on the specific hadron species. Remarkably, the exponent extracted in the prompt photon channel is below those of hadrons, by roughly one unit, close to the conformal limit, $n=4$, at the smallest values of $x_{\perp}$. This observation is understood from the (relative) absence of fragmentation processes and one less power in $\alpha_{s}$, leading to less scaling violation in this channel.

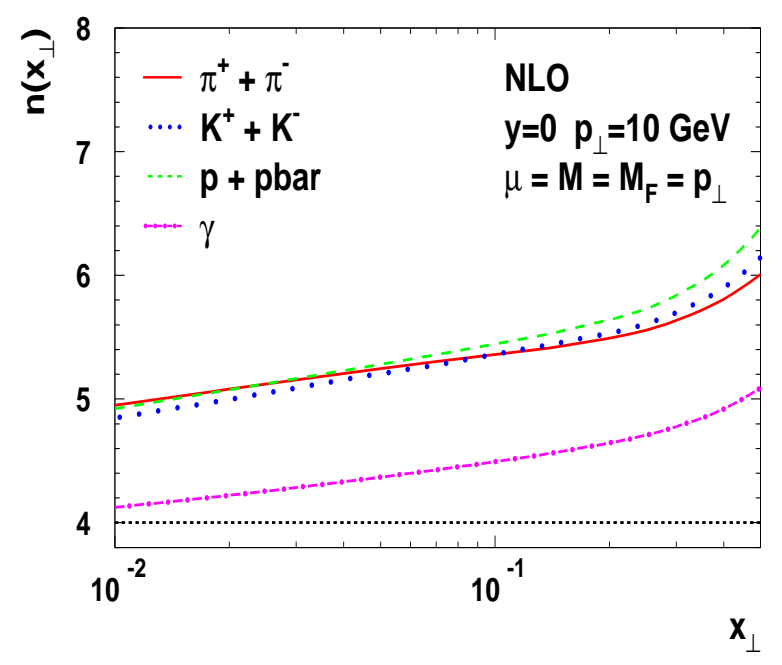

Figure 2: $x_{\perp}$-dependence of $n^{\mathrm{NLO}}$ for $\pi^{ \pm}$(solid line), $K^{ \pm}$(dotted), $p / \bar{p}$ (dashed) and $\gamma$ (dot-dashed), at $p_{\perp}=10 \mathrm{GeV}$.

On the experimental side, the exponent $n^{\exp }$ has been systematically extracted from measurements in hadronic collisions, from fixed-target to collider experiments. It is deduced from the comparison of $x_{\perp}$-spectra at different $\mathrm{CM}$ energies,

$$
n^{\exp }\left(x_{\perp}\right) \equiv-\frac{\ln \left(\sigma^{\mathrm{inv}}\left(x_{\perp}, \sqrt{s_{1}}\right) / \sigma^{\mathrm{inv}}\left(x_{\perp}, \sqrt{s_{2}}\right)\right)}{\ln \left(\sqrt{s_{1}} / \sqrt{s_{2}}\right)}
$$

which is equivalent to (1) at fixed $x_{\perp}$. The data sets include $\pi^{0}$ measurements by the E706 at FNAL [4] and by the PHENIX collaboration at RHIC [5]. At higher energies, the measurements of charged hadrons (or charged tracks) in $p-\bar{p}$ collisions at $\sqrt{s}=630,1800 \mathrm{GeV}$ by CDF [6] and $\sqrt{s}=500,900 \mathrm{GeV}$ by UA1 [7] are included in the analysis. Also considered are prompt photon [8] and jet [9] data obtained by CDF and D0 at $\sqrt{s}=546,630,1800 \mathrm{GeV}$.

\section{Results}

The hadron exponents plotted in Fig. 3 (left) exhibit a clear trend, with a significant rise of $n^{\exp }$ as a function of $x_{\perp}$. Typical values of $n^{\exp }$ are $n^{\exp } \simeq 5-6$ at small $x_{\perp} \simeq 10^{-2}$ while PHENIX data point to a mean value $n^{\exp } \simeq 6.7$ at an intermediate $x_{\perp} \simeq 10^{-1}$. At higher values of $x_{\perp}$, the measurements by PHENIX and E706 reveal an exponent even larger, $n^{\exp } \simeq 8$, confirming the results reported long ago at the ISR. The exponents obtained in the photon and jet channels are strikingly different, showing almost no dependence on $x_{\perp}$. Importantly enough, the values obtained lie only slightly above the conformal limit, $n_{\gamma}^{\exp } \simeq 4.6$ and $n_{\text {jets }}^{\exp } \simeq 4.4$, i.e. several units smaller than the exponents observed for hadrons.

In order to compare properly data and theory, the difference between experimental and theoretical exponents, $\Delta\left(x_{\perp}\right) \equiv n^{\exp }-n^{\mathrm{NLO}}$, is plotted in the right panel of Fig. 3 for hadrons and photons/jets. Note that the error bars include both experimental as well as theoretical errors, added in quadrature. The theoretical uncertainty is estimated from the variation of renormalization/factorization scales from $p_{\perp} / 2$ to $2 p_{\perp}$, as is common practice (the renormalization scale ambiguity can be removed using the methods described in [10]). Fig. 3 (right) indicates that the hadronic exponents extracted experimentally prove significantly above the leadingtwist predictions. The discrepancy is moderate at small $x_{\perp} \sim 10^{-2}, \Delta \simeq 0.5$, but becomes increasingly larger at higher values of $x_{\perp}: \Delta \simeq 1$ at $x_{\perp}=10^{-1}$ and up to $\Delta \simeq 2$ in the largest $x_{\perp}$ region. In contrast, the scaling behavior observed for photons and jets are in very good agreement with the NLO predictions $(\Delta \simeq 0)$.

\section{Discussion}

Part of the discrepancy reported in hadron production data at large $x_{\perp} \sim 1$ could occur because of the appearance of large threshold logarithms, $\ln \left(1-x_{\perp}\right)$, which should be resummed to all orders in perturbation theory [11]. It would therefore be most interesting to investigate whether or not threshold resummation 

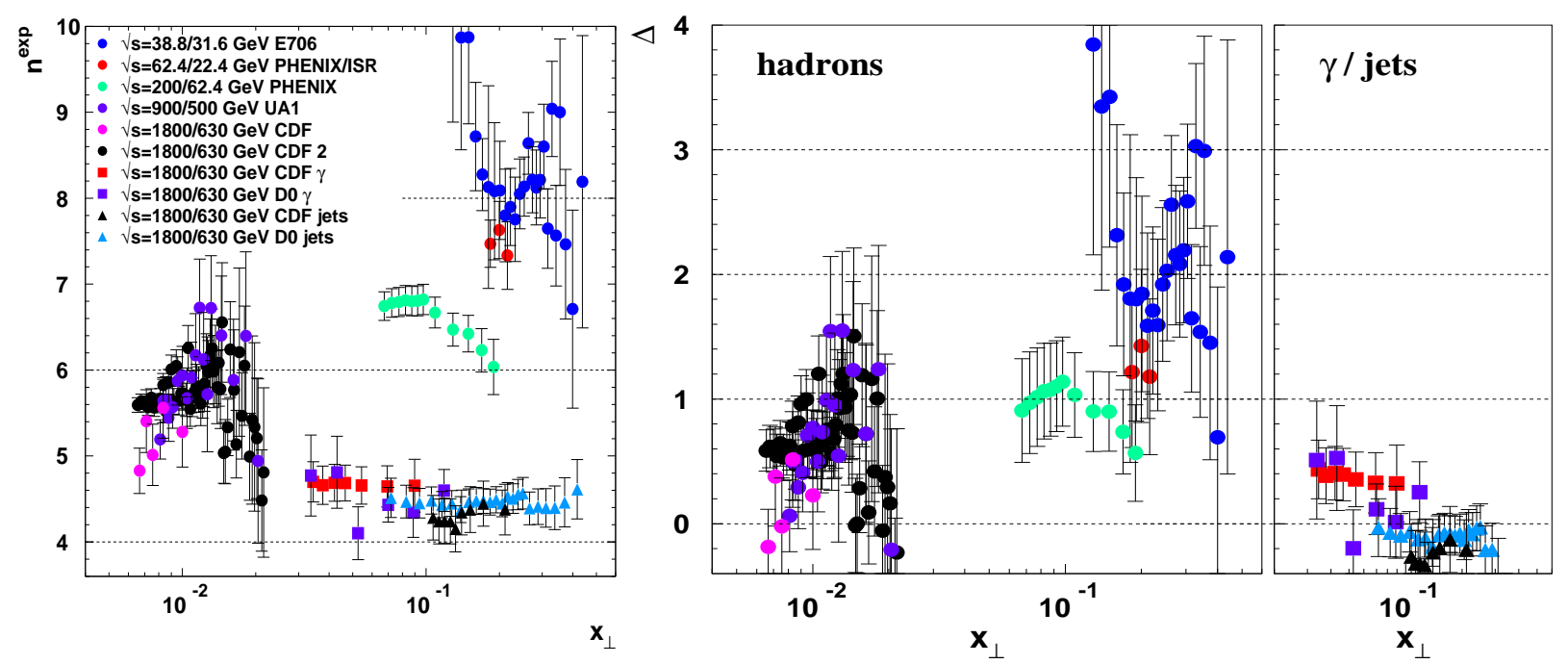

Figure 3: Left: Values of $n^{\exp }$ as a function of $x_{\perp}$ for $h^{ \pm} / \pi^{0}$ (circles), $\gamma$ (squares) and jets (triangles). Right: $\Delta \equiv n^{\exp }-n^{\mathrm{NLO}}$ as a function of $x_{\perp}$, error bars include the experimental and the theoretical uncertainties added in quadrature (see text).

might bring data and theory in agreement. Note however that the discrepancy is also observed at small values of $x_{\perp} \sim 10^{-2}$, where such effects are usually expected to be small.

A natural explanation for the large exponents observed in the hadron channel is the presence of important HT contributions from processes in which the detected hadron is produced directly in the hard subprocess, because of the dimension of the hadron distribution amplitude. In contrast, particles having no hadronic structure like isolated photons and jets are much less sensitive to such HT contributions and should behave closer to LT expectations, as observed. Another piece of evidence for HT effects is the larger exponents for protons than for pions observed at the ISR. As discussed in [2], the difference between the direct proton and pion scaling exponent is $n_{p}-n_{\pi}=2\left(n_{p}=8, n_{\pi}=6\right)$ instead of $n_{p}-n_{\pi} \simeq 0$ at LT. The experimental value obtained from the ISR, $n_{p}-n_{\pi} \simeq 1$, thus reflects the mixture of LT and HT contributions to the total cross section. It has also been noted [12] that the presence of color-transparent HT subprocesses can account for anomalous features of proton production in heavy ion collisions [13].

In order to probe HT contributions more explicitly, let us consider a 2-component model cross section with nominal power dependence

$$
\sigma^{\text {model }}(p p \rightarrow \pi \mathrm{X}) \propto \frac{A\left(x_{\perp}\right)}{p_{\perp}^{4}}+\frac{B\left(x_{\perp}\right)}{p_{\perp}^{6}},
$$

corresponding to the LT $\left(n_{\text {active }}=4\right)$ and HT $\left(n_{\text {active }}=5\right)$ processes, respectively. The actual $p_{\perp}$-exponents are modified by the running coupling and PDF and FF evolution. Assuming that the contributions to $n^{\mathrm{NLO}}-4$ due to pQCD are the same for the LT and HT processes, Eq. (3) gives the effective exponent

$$
\begin{aligned}
n_{\mathrm{eff}}\left(x_{\perp}, p_{\perp}, B / A\right) & \equiv-\frac{\partial \ln \sigma^{\text {model }}}{\partial \ln p_{\perp}}+n^{\mathrm{NLO}}\left(x_{\perp}, p_{\perp}\right)-4 \\
& =\frac{2 B / A}{p_{\perp}^{2}+B / A}+n^{\mathrm{NLO}}\left(x_{\perp}, p_{\perp}\right) .
\end{aligned}
$$

Note that $n_{\text {eff }} \rightarrow n^{\mathrm{NLO}}+2$ for $B / A \rightarrow \infty$. As shown in Fig. 4 (solid line), the LT pion exponent (evaluated at $\left.x_{\perp}=0.2\right)$ slowly decreases with $p_{\perp}$ and reaches $n_{\text {eff }}=4$ as $p_{\perp} \rightarrow \infty$ because of asymptotic freedom. Eq. (4) shows that $n_{\text {eff }}$ depends on the relative strength of HT corrections to the LT cross section, $B / A$. The value $B / A \sim 50 \mathrm{GeV}^{2}\left(\chi^{2} /\right.$ ndf $=0.1$, as compared to $\chi^{2} /$ ndf $=5.2$ when $\left.B / A=0\right)$ is extracted from the data as shown in Fig. 4 (dotted line). However, a somewhat smaller estimate, $B / A \sim 15 \mathrm{GeV}^{2}$, is obtained when all scales are set to $p_{\perp} / 2$ in the QCD calculation. We note that the HT rate for direct processes and therefore $B / A$ are enhanced relative to fragmentation processes since the trigger hadron is produced without any waste of energy; thus the magnitude of the subprocess amplitude is maximized since it is evaluated at the trigger $p_{\perp}$, and the initial momentum fractions $x_{1}$ and $x_{2}$ are evaluated at small values where the PDFs are largest. 


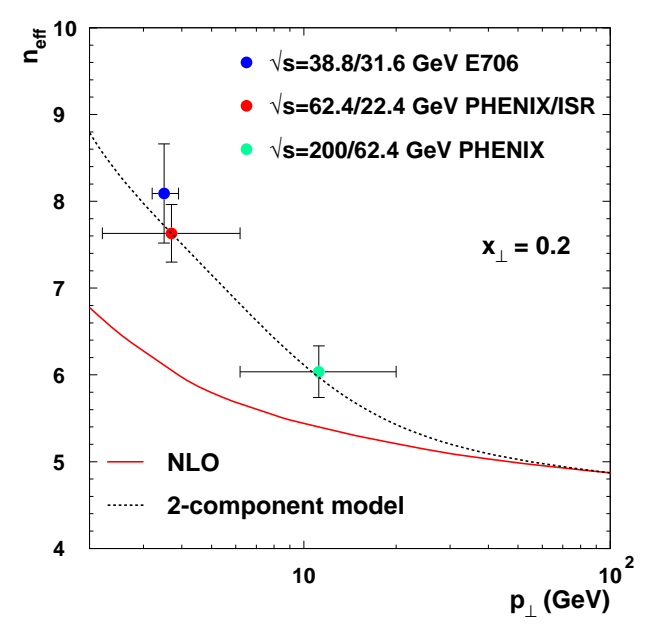

Figure 4: $p_{\perp}$-dependence of $n_{\text {eff }}$ of pions at $x_{\perp}=0.2$ in QCD at NLO (solid line). The dotted line represents a fit based on a two-component model with $B / A=50 \mathrm{GeV}^{2}$, see Eq. (4).

Finally, we discuss the phenomenological consequences of possible HT contributions to hadron production in $p-p$ collisions at RHIC and LHC. In order to obtain qualitative predictions, the difference $\Delta$ between the experimental and the NLO exponent has been fitted to the hadron data currently analyzed. The typical values of $\Delta^{\text {fit }}$ expected at RHIC (taking $\sqrt{s}=200,500 \mathrm{GeV}$ ) and at LHC $(\sqrt{s}=7 \mathrm{TeV}$, compared to $\sqrt{s}=1.8 \mathrm{TeV}$ at Tevatron) are plotted as a function of $x_{\perp}$ in Fig. 5. At RHIC, $\Delta^{\text {fit }}$ is slightly below 1 at small $x_{\perp} \lesssim 5.10^{-2}$ but decreases towards zero at larger $x_{\perp}$. The predictions turn out to be in very good agreement, both in shape and magnitude, with the PHENIX preliminary measurements [14] performed at $\sqrt{s}=500 \mathrm{GeV}$. At LHC, smaller deviations with NLO expectations are expected because of the large values of $p_{\perp}$ probed at high energy: $\Delta^{\text {fit }} \simeq 0.5$ below $x_{\perp}=5 \times 10^{-3}$ and smaller above.

We also point that the use of isolation cuts, usually applied for prompt photons, will strongly suppress LT processes. As a consequence the scaling exponents of isolated hadrons (i.e. with small hadronic activity in their vicinity) are expected to be somewhat larger than those in the inclusive channel. Conversely, using isolated hadrons as a signal for Higgs production or new physics scenarios [15] might be confused by the presence of direct hadron production processes.

We thank A. Bazilevsky for providing us with Fig. 5. FA thanks CERN-TH for hospitality. SJB was supported by the Department of Energy under contract DE-AC02-76SF00515. DSH was supported by the In-

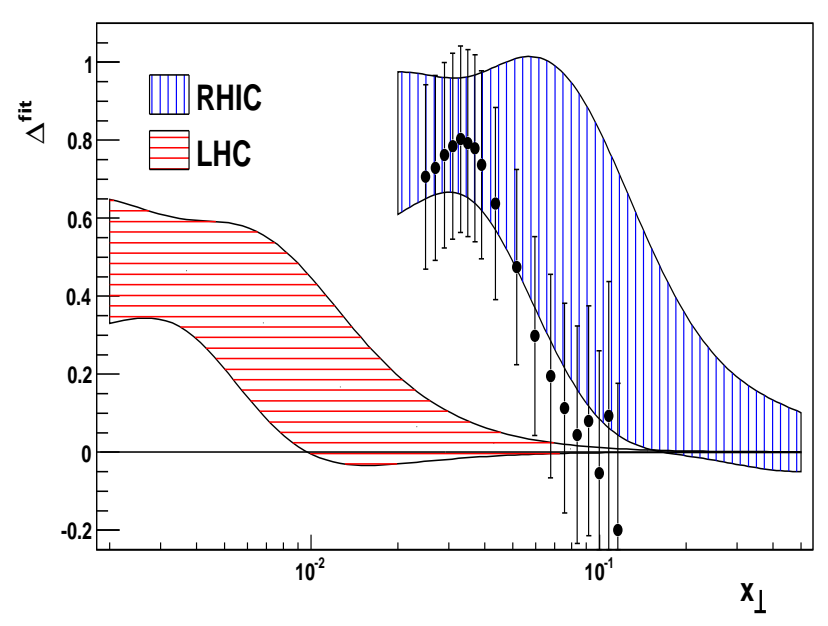

Figure 5: Predicted difference between the experimental and NLO scaling exponent at RHIC $(\sqrt{s}=200,500 \mathrm{GeV})$ and the LHC $(\sqrt{s}=$ $7 \mathrm{TeV}$ as compared to $\sqrt{s}=1.8 \mathrm{TeV}$ ), compared to PHENIX preliminary measurements.

ternational Cooperation Program of the KICOS and the Korea Research Foundation Grant (KRF-2008-313C00166). AMS was supported by the Department of Energy under contract DE-AC02-98CH10886.

\section{References}

[1] See e.g. S. J. Brodsky, G. R. Farrar, Phys. Rev. Lett. 31 (1973) 1153

[2] F. Arleo, S. J. Brodsky, D.S. Hwang, A. M. Sickles, Phys. Rev. Lett. 105 (2010) 062002

[3] P. Aurenche et al., Eur. Phys. J. C 9 (1999) 107; ibid., Eur. Phys. J. C 13 (2000) 347; Z. Kunszt and D. E. Soper, Phys. Rev. D 46 (1992) 192

[4] L. Apanasevich et al., Phys. Rev. D 68 (2003) 052001

[5] A. Adare et al., Phys. Rev. D 76 (2007) 051106; ibid., 79 (2009) 012003

[6] F. Abe et al., Phys. Rev. Lett. 61 (1988) 1819; D. E. Acosta et al., Phys. Rev. D 65 (2002) 072005

[7] C. Albajar et al., Nucl. Phys. B335 (1990) 261

[8] D. E. Acosta et al., Phys. Rev. D 65 (2002) 112003; B. Abbott et al., Phys. Rev. Lett. 84 (2000) 2786; V. M. Abazov et al., Phys. Rev. Lett. 87 (2001) 251805

[9] F. Abe et al., Phys. Rev. Lett. 70 (1993) 1376; B. Abbott et al., Phys. Rev. D 64 (2001) 032003

[10] M. Binger, S.J. Brodsky, Phys. Rev. D 74 (2006) 054016

[11] See e.g. D. de Florian and W. Vogelsang, Phys. Rev. D 71 (2005) 114004.

[12] S. J. Brodsky, A. Sickles, Phys. Lett. B 668 (2008) 111

[13] S. S. Adler et al., Phys. Rev. Lett. 91 (2003) 172301

[14] A. Bazilevsky, talk at APS Meeting 2010, http://meetings . aps.org/Meeting/APR10

[15] A. Nisati (ATLAS), and Y. Sirois (CMS), private communication. 